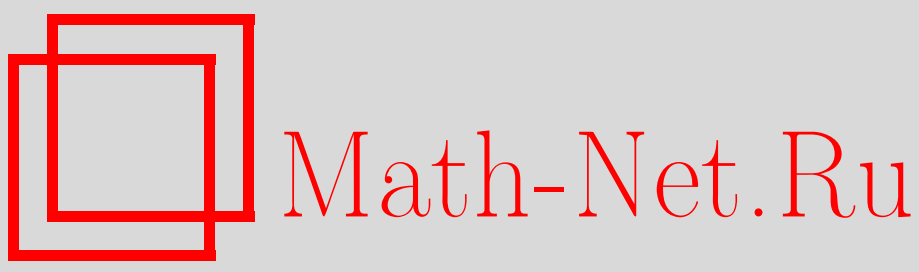

E. V. Sokolov, E. A. Tumanova, The root-class residuality of tree products with central amalgamated subgroups, Sibirsk. Mat. Zh., 2020, Volume 61, Number 3, 692-702

DOI: https://doi.org/10.33048/smzh.2020.61.318

Use of the all-Russian mathematical portal Math-Net.Ru implies that you have read and agreed to these terms of use http://www . mathnet.ru/eng/agreement

Download details:

IP: 34.229 .45 .116

April 26, 2023, 11:06:17

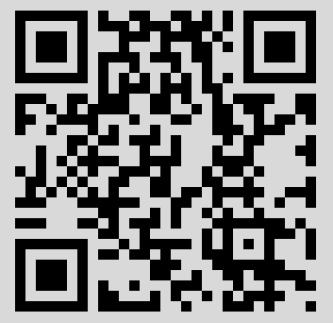


Сибирский математический журнал Май-июнь, 2020. Том 61, № 3

УДК 512.543

\title{
ОБ АППРОКСИМИРУЕМОСТИ КОРНЕВЫМИ КЛАССАМИ ДРЕВЕСНЫХ ПРОИЗВЕДЕНИЙ С ЦЕНТРАЛЬНЫМИ ОБЪЕДИНЕННЫМИ ПОДГРУППАМИ
}

\author{
Е. В. Соколов, Е. А. Туманова
}

\begin{abstract}
Аннотация. Пусть $\mathscr{C}$ - корневой класс групп, $P$ - древесное произведение, каждая объединенная подгруппа которого лежит в центрах соответствующих вершинных групп. В статье указаны некоторые достаточные условия $\mathscr{C}$-аппроксимируемости группы $P$. Доказано, в частности, что древесное произведение разрешимых групп ограниченной ступени разрешимости с центральными объединенными подгруппами аппроксимируется разрешимыми группами.
\end{abstract}

DOI 10.33048/smzh.2020.61.318

Ключевые слова: древесное произведение, финитная аппроксимируемость, аппроксимируемость конечными $p$-группами, аппроксимируемость разрешимыми группами, аппроксимируемость корневыми классами групп.

\section{$\S 1$. Введение}

Напомним, что группа $X$ называется аппроксимируемой классом групп $\mathscr{C}$ или, короче, $\mathscr{C}$-аппроксимируемой, если для каждого ее неединичного элемента найдется гомоморфизм группы $X$ на группу из класса $\mathscr{C}$ ( $\mathscr{C}$-группу), при котором образ этого элемента по-прежнему отличен от 1 . Как следует из названия, в данной статье изучается аппроксимируемость корневыми классами групп.

Понятие корневого класса введено Грюнбергом в 1957 г. [1]. К этому времени результатов об аппроксимируемости конкретными классами групп было известно уже достаточно много и доказательства части из них содержали весьма похожие схемы рассуждений. Новое понятие позволяло провести эти рассуждения однократно, используя лишь общие свойства аппроксимирующих классов групп.

Согласно данному Грюнбергом определению класс групп $\mathscr{C}$ является корневым, если он замкнут относительно взятия подгрупп и прямых произведений конечного числа сомножителей, а также удовлетворяет следующему условию, которое теперь обычно называют условием Грюнберга: если $1 \leq Z \leq Y \leq X-$ субнормальный ряд некоторой группы $X$, факторы $X / Y$ и $Y / Z$ которого принадлежат классу $\mathscr{C}$, то в группе $X$ найдется нормальная подгруппа $T$ такая, что $T \leq Z$ и $X / T \in \mathscr{C}$. Последняя, наиболее странная часть данного определения, носит чисто утилитарный характер: она позволяет доказать весьма полезное

Работа выполнена при финансовой поддержке Российского фонда фундаментальных исследований (код проекта 18-31-00187).

(c) 2020 Соколов Е. В., Туманова Е. А. 
и часто используемое утверждение о том, что если $\mathscr{C}-$ корневой класс групп, то произвольное расширение $\mathscr{C}$-аппроксимируемой группы при помощи группы из класса $\mathscr{C}$, в свою очередь, является $\mathscr{C}$-аппроксимируемой группой. Вместе с тем, глядя на условие Грюнберга, достаточно сложно понять, что же представляют собой корневые классы групп в целом.

Равносильное, более простое, определение корневого класса приводится в [2], где доказано, что класс групп корневой тогда и только тогда, когда он замкнут относительно взятия подгрупп и расширений, а также вместе с любыми двумя группами $X$ и $Y$ содержит декартово произведение вида $\prod_{y \in Y} X_{y}$, где $X_{y}$ - изоморфная копия группы $X$ для каждого $y \in Y$. Из приведенного определения легко следует, что пересечение любого числа корневых классов снова корневой класс. Поскольку класс всех групп без кручения корневой, отсюда вытекает, в частности, что если $\mathscr{C}$ - корневой класс, то множество всех $\mathscr{C}$-групп без кручения также является корневым классом (это замечание будет несколько раз использоваться в дальнейшем).

Примерами корневых классов, аппроксимируемость которыми чаще всего исследуется в литературе, являются классы всех конечных групп, конечных $p$-групп (где $p$ - простое число), периодических $\pi$-групп конечного периода (где $\pi$ - непустое множество простых чисел), разрешимых групп, а также всевозможные их пересечения. Класс всех нильпотентных групп, также нередко выступающий в качестве аппроксимирующего, не замкнут относительно взятия расширений и потому корневым не является. Однако для некоторых групп нильпотентную аппроксимируемость удается полностью исследовать, пользуясь лишь критерием аппроксимируемости конечными $p$-группами (см., например, [3]). Таким образом, результаты об аппроксимируемости корневыми классами могут оказаться полезными и в этом случае.

При изучении аппроксимируемости корневым классом $\mathscr{C}$ любой свободной конструкции групп основным является вопрос о наследовании данной конструкцией свойства $\mathscr{C}$-аппроксимируемости от групп, из которых она построена. В полном объеме ответить на него удалось только в случае свободного произведения произвольного семейства групп (см. предложение 3 ниже). Для более сложно устроенных конструкций таких, как свободное произведение групп с объединенной подгруппой и HNN-расширение, общее описание условий, при которых указанное наследование имеет место, неизвестно. Более того, анализируя формулировки полученных к настоящему времени результатов об аппроксимируемости этих конструкций конкретными корневыми классами групп (см., например, [4-9]), можно с высокой долей уверенности утверждать, что решения, столь же простого и универсального, как для обычного свободного произведения групп, здесь найти не удастся. Как следствие, аппроксимируемость подобных свободных конструкций изучают при различных дополнительных ограничениях, накладываемых на группы, из которых они построены, на объединенные или связанные подгруппы, а также на аппроксимирующий класс. В последние годы на этом пути было получено достаточно много результатов об аппроксимируемости уже не каким-то конкретным, а произвольным корневым классом групп, удовлетворяющим, возможно, некоторым дополнительным условиям (см., например, $[2,10-21])$. Следует заметить, что отправной точкой для всех этих исследований послужило сделанное Д. Н. Азаровым замечание о том, что любая свободная группа аппроксимируется каждым нетривиальным (т. е. содержащим хотя бы одну неединичную группу) корневым классом 
групп [10].

В настоящей работе изучается аппроксимируемость корневыми классами древесных произведений, реберные подгруппы которых содержатся в центрах вершинных групп (определение конструкции древесного произведения приводится в $\S 2$ ). Основная идея состоит в рассмотрении гомоморфизма древесного произведения на аналогичную конструкцию, построенную на основе прямого, а не свободного произведения групп. Впервые данный подход использовался в [11] при изучении аппроксимируемости корневыми классами свободного произведения двух групп с центральными объединенными подгруппами.

Сто́ит отметить, что аппроксимируемость древесных произведений исследовалась в основном в предположении, что все реберные подгруппы циклические или имеют конечные индексы в вершинных группах (см., например, $[4,22-$ 24]). Для древесного произведения с центральными реберными подгруппами известно лишь несколько утверждений:

а) достаточное условие финитной аппроксимируемости, которое, скорее всего, не может быть сколько-нибудь существенно усилено [25, следствие 3.9];

б) два критерия аппроксимируемости конечными $p$-группами, полученные в предположении, что все вершинные группы конечные абелевы или свободные абелевы конечного ранга [26, теорема 13, следствие 15.2];

в) два достаточных условия аппроксимируемости разрешимыми группами [27, теорема 3; 28, теорема 3].

Доказанные ниже теоремы 1,2 и следствия 1,2 в определенной мере дополняют перечисленные результаты. В частности, утверждение 1 следствия 1 обобщает упомянутые выше теоремы из $[27,28]$ в части, касающейся аппроксимируемости разрешимыми группами.

\section{$\S 2$. Формулировка результатов}

Пусть $T=(V, E)$ - непустое, не обязательно конечное дерево с множеством вершин $V$ и множеством ребер $E$ (все вводимые в данном параграфе обозначения и предположения остаются неизменными до конца статьи). Сопоставляя каждой вершине $v \in V$ некоторую группу $G_{v}$ и каждому ребру $e=\{v, w\} \in E$ группу $H_{e}$ и инъективные гомоморфизмы $\varphi_{e, v}: H_{e} \rightarrow G_{v}, \varphi_{e, w}: H_{e} \rightarrow G_{w}$, получим граф груnп, который далее будем обозначать той же буквой $T$. Группы $G_{v}(v \in V), H_{e}(e \in E)$, подгруппы $H_{e, v}=H_{e} \varphi_{e, v}$ и гомоморфизмы $\varphi_{e, v}(e \in E$, $v \in e)$ будем называть соответственно вериинными и реберными группами, реберными подгруппами и реберными гомоморфизмами.

Рассмотрим группы

$$
\begin{gathered}
\operatorname{FTP}(T)=\left\langle G_{v}(v \in V) ; H_{e, v}=H_{e, w}(e=\{v, w\} \in E)\right\rangle, \\
\operatorname{DTP}(T)=\left\langle G_{v}(v \in V) ; H_{e, v}=H_{e, w}(e=\{v, w\} \in E),\right. \\
\left.\left[G_{u}, G_{v}\right]=1(u, v \in V, u \neq v)\right\rangle,
\end{gathered}
$$

образующими которых являются образующие групп $G_{v}(v \in V)$, а определяющими соотношениями - определяющие соотношения групп $G_{v}(v \in V)$, всевозможные соотношения вида $h \varphi_{e, v}=h \varphi_{e, w}$, где $e=\{v, w\} \in E, h \in H_{e}$, а также (в случае группы $\operatorname{DTP}(T))$ соотношения вида $\left[g_{u}, g_{v}\right]=1$, где $u, v \in V, u \neq v$, $g_{u}$ - произвольный образующий группы $G_{u}, g_{v}$ - произвольный образующий группы $G_{v}$. 
Группа $\operatorname{FTP}(T)$ называется древесным произведением групп $G_{v}(v \in V)$ [29]. Очевидно, что она является фактор-группой свободного произведения групп $G_{v}$ по нормальному замыканию множества элементов

$$
\left\{h \varphi_{e, v}\left(h \varphi_{e, w}\right)^{-1} \mid e=\{v, w\} \in E, h \in H_{e}\right\} .
$$

Поскольку группа $\operatorname{DTP}(T)$ представляет собой точно такую же фактор-группу прямого произведения групп $G_{v}$, ее естественно назвать прямым древесным произведением групп $G_{v}(v \in V)$.

Известно (см. [29, теорема 1]), что для каждой вершины $v \in V$ тождественное отображение образующих группы $G_{v}$ в группу $\mathrm{FTP}(T)$ продолжаемо до инъективного гомоморфизма и потому все группы $G_{v}(v \in V)$ можно считать подгруппами группы $\operatorname{FTP}(T)$. Если для любых $e \in E, v \in e$ подгруппа $H_{e, v}$ лежит в центре группе $G_{v}$, тем же свойством обладает и группа $\mathrm{DTP}(T)$ (см. предложение 6 ниже). Этот факт играет важную роль в доказательстве основных результатов настоящей работы, формулировки которых приводятся ниже.

Теорема 1. Пусть $T$ - произвольное дерево, $\mathscr{C}$ - нетривиальный корневой класс групп, для любых $e \in E, v \in e$ подгруппа $H_{e, v}$ лежит в центре группы $G_{v}$ и $\operatorname{DTP}(T) \in \mathscr{C}$. Тогда группа $\operatorname{FTP}(T) \mathscr{C}$-аппроксимируема.

Конкретным примером применения теоремы 1 может служить

Следствие 1. Пусть $T$ - произвольное дерево и для любых $e \in E, v \in e$ подгруппа $H_{e, v}$ лежит в центре группы $G_{v}$. Если все группы $G_{v}(v \in V)$ разрешимы и их ступени разрешимости ограничены в совокупности, то справедливы следующие утверждения.

1. Группа $\operatorname{FTP}(T)$ аппроксимируется разрешимыми группами.

2. Если все группы $G_{v}(v \in V)$ не имеют кручения и для любых $e \in E, v \in e$ подгруппа $H_{e, v}$ изолирована в группе $G_{v}$, то группа $\operatorname{FTP}(T)$ аппроксимируется разрешимыми группами без кручения.

3. Если все группы $G_{v}(v \in V)$ являются периодическими $\pi$-группами конечного периода для некоторого множества простых чисел $\pi$, то группа $\operatorname{FTP}(T)$ аппроксимируется периодическими разрешимыми $\pi$-группами конечного периода.

В случае, когда дерево конечно и класс $\mathscr{C}$ замкнут относительно взятия гомоморфных образов, имеет место более сильное, нежели теорема 1 , утверждение, обобщающее теорему 2 из [11].

Теорема 2. Пусть $T-$ конечное дерево, $\mathscr{C}-$ нетривиальный корневой класс групп, замкнутый относительно взятия фактор-групп, и для любых $e \in E$, $v \in e$ подгруппа $H_{e, v}$ лежит в центре группы $G_{v}$. Пусть также каждая вершинная группа $G_{v} \mathscr{C}$-аппроксимируема и обладает гомоморфизмом $\sigma_{v}$ на группу из класса $\mathscr{C}$, инъективным на всех реберных подгруппах, лежащих в $G_{v}$. Тогда существует гомоморфизм $\sigma$ группы $\mathrm{FTP}(T)$ на группу из класса $\mathscr{C}$, продолжающий гомоморфизмы $\sigma_{v}(v \in V)$, и группа $\operatorname{FTP}(T) \mathscr{C}$-аппроксимируема.

Пусть в дополнение к условиям, перечисленным выше, для любых $e \in E$, $v \in e$ группа $G_{v} \sigma_{v}$ не имеет кручения и подгруппа $H_{e, v} \sigma_{v}$ изолирована в ней. Тогда образ гомоморфизма $\sigma$ можно считать группой без кручения, и если каждая вершинная группа $G_{v}$ аппроксимируется $\mathscr{C}$-группами без кручения, то и группа $\operatorname{FTP}(T)$ аппроксимируется $\mathscr{C}$-группами без кручения.

В качестве комментария к формулировке теоремы 2 отметим, что, как установлено в [20], для многих корневых классов групп существование гомоморфизма группы $\operatorname{FTP}(T)$ на группу из класса $\mathscr{C}$, инъективного на каждой реберной 
подгруппе, является более сильным условием, нежели $\mathscr{C}$-аппроксимируемость. При этом именно наличие такого гомоморфизма, а не просто $\mathscr{C}$-аппроксимируемость, зачастую оказывается необходимым для исследования аппроксимируемости свободных конструкций групп корневыми классами, содержащими бесконечные группы (см., например, $[13,15,21])$.

Следствие 2. Пусть $T-$ конечное дерево, $\mathscr{C}-$ нетривиальный корневой класс групп, замкнутый относительно взятия фактор-групп, и для любых $e \in$ $E, v \in e$ подгруппа $H_{e, v}$ лежит в центре группы $G_{v} . \quad \Gamma$ руппа $\operatorname{FTP}(T) \mathscr{C}$-аппроксимируема, если для любой вершины $v \in V$ выполняется хотя бы одно из следующих трех условий:

1) группа $G_{v}$ принадлежит классу $\mathscr{C}$;

2) группа $G_{v} \mathscr{C}$-аппроксимируема и все лежащие в ней подгруппы $H_{e, v}$ конечны;

3) группа $G_{v}$ аппроксимируется $\mathscr{C}$-группами без кручения и все лежащие в ней подгруппы $H_{e, v}$ имеют конечный ранг.

Оставшаяся часть статьи посвящена доказательству сформулированных выше теорем и следствий.

\section{§ 3. Доказательства теорем 1 и 2}

Пусть $T$ - произвольное дерево. Предположим, что для всякой вершины $v \in V$ зафиксирована нормальная подгруппа $R_{v}$ группы $G_{v}$, тривиально пересекающаяся с каждой подгруппой $H_{e, v}$, где $v \in e$. Тогда композиция $\overline{\varphi_{e, v}}$ отображения $\varphi_{e, v}$ и естественного гомоморфизма группы $G_{v}$ на фактор-группу $\overline{G_{v}}=G_{v} / R_{v}$ является вложением для любых $e \in E, v \in e$. Следовательно, наряду с графом групп $T$ можно рассмотреть граф групп $T_{\mathscr{R}}$, где $\mathscr{R}=\left\{R_{v} \mid v \in V\right\}$, с теми же множествами вершин и ребер $V$ и $E$, элементам которых сопоставлены группы $\overline{G_{v}}(v \in V), H_{e}(e \in E)$ и определенные выше вложения $\overline{\varphi_{e, v}}(e \in E$, $v \in e)$. Представление группы $\operatorname{FTP}\left(T_{\mathscr{R}}\right)$ отличается от представления группы $\operatorname{FTP}(T)$ только дополнительными определяющими словами, задающими порождающие подгрупп $R_{v}(v \in V)$. Поэтому справедливо

Предложение 1. Тождественное отображение образующих группы FTP $(T)$ в группу $\operatorname{FTP}\left(T_{\mathscr{R}}\right)$, будучи продолженным до отображения слов, переводит все определяющие соотношения группы $\operatorname{FTP}(T)$ в равенства, верные в группе $\operatorname{FTP}\left(T_{\mathscr{R}}\right)$, и, следовательно, определяет сюръективный гомоморфизм $\rho$ первой на вторую, действие которого на вершинных группах $G_{v}$ совпадает с действием естественных гомоморфизмов $G_{v} \rightarrow \overline{G_{v}}$. Ядро гомоморфизма $\rho$ представляет собой нормальное замыкание в группе $\operatorname{FTP}(T)$ объединения подгрупп $R_{v}$ $(v \in V)$.

Предложение 2. Пусть $T$ - конечное дерево и $N-$ нормальная подгруппа группы $\mathrm{FTP}(T)$, тривиально пересекающаяся с подгруппами $H_{e, v}$ для всех $e \in E, v \in e$. Тогда подгруппа $N$ раскладывается в свободное произведение семейства групп, каждая из которых свободна или изоморфна $N \cap G_{v}$ для некоторой вершины $v \in V$.

ДокАЗАТЕЛЬСтво. Воспользуемся индукцией по количеству $n$ ребер в графе $T$. Если $n=0$, требуемое утверждение очевидно. Поэтому будем считать далее, что $n>0$ и для всех деревьев с числом ребер, меньшим $n$, доказываемое предложение имеет место. 
Пусть $e=\{v, w\}$ - произвольное ребро графа $T, T_{1}$ и $T_{2}$ - компоненты связности графа, который получается из $T$ путем удаления ребра $e$. Тогда согласно теореме 1 из [29] группа $\operatorname{FTP}(T)$ представляет собой свободное произведение групп $\operatorname{FTP}\left(T_{1}\right)$ и $\operatorname{FTP}\left(T_{2}\right)$ с объединенными подгруппами $H_{e, v}$ и $H_{e, w}$. В силу теоремы 5 из [29] подгруппа $N$ раскладывается в свободное произведение некоторой свободной группы и семейства групп, каждая из которых изоморфна $N \cap \operatorname{FTP}\left(T_{1}\right)$ или $N \cap \operatorname{FTP}\left(T_{2}\right)$. Последние две группы по индуктивному предположению, в свою очередь, раскладываются в свободные произведения семейств групп, каждая из которых свободна или изоморфна $N \cap G_{v}$ для некоторой вершины $v \in V$. Таким образом, подгруппа $N$ имеет требуемое разложение.

В приводимом ниже предложении собраны известные результаты об аппроксимируемости корневыми классами, необходимые для дальнейших рассуждений.

Предложение 3. Пусть $\mathscr{C}$ - нетривиальный корневой класс групп. Тогда имеют место следующие утверждения.

1. Каждая свободная группа аппроксимируется классом $\mathscr{C}$ [10, теорема 1].

2. Свободное произведение произвольного семейства $\mathscr{C}$-аппроксимируемых групп, в свою очередь, аппроксимируется классом $\mathscr{C}[1$, теорема $4.1 ; 10]$.

3. Любое расширение $\mathscr{C}$-аппроксимируемой группы при помощи $\mathscr{C}$-группы аппроксимируется классом $\mathscr{C}[1$, лемма 1.5$]$.

Следующие два предложения составляют основную часть доказательства теорем 1 и 2.

Предложение 4. Пусть $T$ - конечное дерево, $\mathscr{C}$ - нетривиальный корневой класс групп и для каждой вершины $v \in V$ группа $G_{v} \mathscr{C}$-аппроксимируема. Если существует гомоморфизм $\sigma$ группы $\mathrm{FTP}(T)$ на группу из класса $\mathscr{C}$, инъективный на всех подгруппах $H_{e, v}(e \in E, v \in e)$, то группа $\operatorname{FTP}(T) \mathscr{C}$-аппроксимируема.

ДокАзАтЕльство. Согласно предложению 2 подгруппа $N=\operatorname{ker} \sigma$ раскладывается в свободное произведение семейства групп, каждая из которых свободна или изоморфна $N \cap G_{v}$ для некоторой вершины $v \in V$. Свободная группа аппроксимируется классом $\mathscr{C}$ в силу утверждения 1 предложения 3 , группа $N \cap G_{v}$ - как подгруппа $\mathscr{C}$-аппроксимируемой группы $G_{v}$. Следовательно, подгруппа $N$ аппроксимируется классом $\mathscr{C}$ согласно утверждению 2 предложения 3 и группа $\operatorname{FTP}(T)$ оказывается расширением $\mathscr{C}$-аппроксимируемой группы $N$ при помощи $\mathscr{C}$-группы $\mathrm{FTP}(T) / N$. Такое расширение аппроксимируется классом $\mathscr{C}$ ввиду утверждения 3 предложения 3.

Далее, если $v$ - произвольная вершина дерева $T$, то через $H_{v}$ будем обозначать подгруппу группы $G_{v}$, порожденную всеми подгруппами семейства $\left\{H_{e, v} \mid\right.$ $v \in e\}$ (т. е. всеми реберными подгруппами, содержащимися в группе $G_{v}$ ).

Предложение 5. Пусть $T-$ произвольное дерево, $\mathscr{C}-$ нетривиальный корневой класс групп и для каждой вершины $v \in V$ группа $G_{v} \mathscr{C}$-аппроксимируема. Если существует гомоморфизм $\sigma$ группы $\operatorname{FTP}(T)$ на группу из класса $\mathscr{C}$, инъективный на всех подгруппах $H_{v}(v \in V)$, то группа $\operatorname{FTP}(T) \mathscr{C}$-аппроксимируема.

ДокАЗАТЕЛЬСтво. Пусть $\omega-$ произвольное слово, определяющее неединичный элемент $g$ группы $\operatorname{FTP}(T)$, и $T_{g}=\left(V_{g}, E_{g}\right)$ - некоторое конечное поддерево графа $T$, вершинные группы которого содержат все образующие, входящие 
в $\omega$. Без потери общности можно считать, что из каждой вершины поддерева $T_{g}$ выходит лишь конечное число ребер.

В самом деле, если это не так, модифицируем граф $T$ следующим образом. Для всякой вершины $v \in V_{g}$ добавим к графу $T$ новую вершину $v^{+}$и ребро $e_{v}$, соединяющее $v$ и $v^{+}$. Каждое ребро $\{v, w\} \in E_{g}$ удалим, заменив ребром $\left\{v^{+}, w^{+}\right\}$. Вершине $v \in V_{g}$ вместо группы $G_{v}$ поставим в соответствие группу $H_{v}$, вершине $v^{+}$- изоморфную копию $G_{v^{+}}$группы $G_{v}$, обозначив изоморфизм $G_{v} \rightarrow G_{v^{+}}$через $\alpha_{v}$. Ребру $e^{+}=\left\{v^{+}, w^{+}\right\}$, соответствующему ребру $e=\{v, w\} \in E_{g}$, сопоставим группу $H_{e}$ и гомоморфизмы $\varphi_{e^{+}, v^{+}}=\varphi_{e, v} \alpha_{v}$, $\varphi_{e^{+}, w^{+}}=\varphi_{e, w} \alpha_{w}$, peбру $e_{v}\left(v \in V_{g}\right)-$ группу $H_{v}$ и гомоморфизмы $\varphi_{e_{v}, v}: H_{v} \rightarrow$ $H_{v}, \varphi_{e_{v}, v^{+}}: H_{v} \rightarrow G_{v^{+}}$, первый из которых является тождественным отображением, а второй - ограничением на подгруппу $H_{v}$ изоморфизма $\alpha_{v}$. Поскольку для любых $e \in E, v \in e$ образ гомоморфизма $\varphi_{e, v}$ содержится в $H_{v}$, можно считать, что всем ребрам, соединяющим некоторую вершину из $V_{g}$ с некоторой вершиной, не принадлежащей $V_{g}$, в новом графе соответствуют те же гомоморфизмы, что и в исходном графе $T$. Модифицированный таким образом граф групп обозначим через $T^{+}$, а его поддерево, порожденное множеством вершин $\left\{v^{+} \mid v \in V_{g}\right\}$, - через $T_{g}^{+}$.

Легко видеть, что отображение образующих группы $\operatorname{FTP}\left(T^{+}\right)$в группу $\operatorname{FTP}(T)$, переводящее для каждой вершины $v \in V_{g}$ образующий $x$ группы $G_{v^{+}}$ в элемент $x \alpha_{v}^{-1}$ группы $G_{v}$ и действующее на остальных образующих тождественно, определяет изоморфизм $\alpha$ группы $\operatorname{FTP}\left(T^{+}\right)$на группу $\operatorname{FTP}(T)$. Так как для любой вершинной группы $X$ графа $T^{+}$этот изоморфизм отображает все лежащие в $X$ реберные подгруппы в некоторую подгруппу $H_{v}$ группы $\operatorname{FTP}(T)$, то композиция $\alpha \sigma$ представляет собой гомоморфизм группы $\operatorname{FTP}\left(T^{+}\right)$ на группу из класса $\mathscr{C}$, удовлетворяющий условию предложения. Кроме того, $g \alpha^{-1} \in \operatorname{FTP}\left(T_{g}^{+}\right)$, но каждая вершина дерева $T_{g}^{+}$соединяется ребрами только с вершинами из конечного множества $\left\{v^{+} \mid v \in V_{g}\right\} \cup\left\{v \mid v \in V_{g}\right\}$.

Итак, далее можно считать, что множество $E^{\prime}$ всех ребер графа $T$, каждое из которых соединяет некоторую вершину из $V_{g}$ с вершиной, не принадлежащей $V_{g}$, конечно. Поэтому граф, получающийся из $T$ в результате удаления ребер, входящих в $E^{\prime}$, имеет конечное число компонент связности $T_{0}=T_{g}$, $T_{1}, \ldots, T_{n}$.

Так как $T$ - ациклический граф, то для любых $i, j \in\{0, \ldots, n\}, i \neq j$, в нем имеется не более одного ребра, связывающего некоторую вершину из $T_{i}$ с некоторой вершиной из $T_{j}$. Поэтому можно рассмотреть граф $T^{*}$ с множеством вершин $V^{*}=\left\{T_{i} \mid 0 \leq i \leq n\right\}$ и множеством ребер $E^{*}$, в котором $\left\{T_{i}, T_{j}\right\} \in E^{*}$ тогда и только тогда, когда существует ребро $e \in E$, ведущее из $T_{i}$ в $T_{j}$. Coпоставим вершине $T_{i} \in V^{*}$ группу $\operatorname{FTP}\left(T_{i}\right)$, а ребру $\left\{T_{i}, T_{j}\right\} \in E^{*}$, соответствующему ребру $e=\{v, w\} \in E,-$ группу $H_{e}$ и вложения $\varphi_{e, v}^{*}, \varphi_{e, w}^{*}$, являющиеся композициями отображений $\varphi_{e, v}, \varphi_{e, w}$ и естественных вложений групп $G_{v}$ и $G_{w}$ в группы $\operatorname{FTP}\left(T_{i}\right)$ и $\operatorname{FTP}\left(T_{j}\right)$ соответственно. Легко видеть, что граф $T^{*}$ является деревом (более точно, звездой с центром $T_{0}=T_{g}$ ), а группы $\operatorname{FTP}\left(T^{*}\right)$ и $\operatorname{FTP}(T)$ имеют одинаковые представления и, в частности, изоморфны. Поэтому далее вместо группы $\operatorname{FTP}(T)$ будем рассматривать группу $\operatorname{FTP}\left(T^{*}\right)$, считая, что гомоморфизм $\sigma$ задан на ней.

Пусть $N=\operatorname{ker} \sigma, R_{0}=1$ и $R_{i}=N \cap \operatorname{FTP}\left(T_{i}\right)$ при $i \geq 1$. Тогда $R_{i}-$ нормальная подгруппа группы $\operatorname{FTP}\left(T_{i}\right)$ для каждого $i \geq 0$ и $\operatorname{FTP}\left(T_{i}\right) / R_{i} \in \mathscr{C}$ 
при $i \geq 1$, поскольку

$$
\operatorname{FTP}\left(T_{i}\right) / R_{i}=\operatorname{FTP}\left(T_{i}\right) /\left(N \cap \operatorname{FTP}\left(T_{i}\right)\right) \cong \operatorname{FTP}\left(T_{i}\right) N / N \leq \operatorname{FTP}(T) / N
$$

и $\operatorname{FTP}(T) / N \in \mathscr{C}$. Заметим также, что при $i=0$ группа $\operatorname{FTP}\left(T_{i}\right) / R_{i} \cong \operatorname{FTP}\left(T_{g}\right)$ $\mathscr{C}$-аппроксимируема в силу предложения 4.

Так как подгруппа $N$ тривиально пересекается с группами $H_{v}(v \in V)$, а значит, и со всеми реберными подгруппами группы $\operatorname{FTP}\left(T^{*}\right)$, можно рассмотреть граф групп $T_{\mathscr{R}}^{*}$, где $\mathscr{R}=\left\{R_{i} \mid 0 \leq i \leq n\right\}$. Согласно предложению 1 тождественное отображение образующих группы $\operatorname{FTP}\left(T^{*}\right)$ в группу $\operatorname{FTP}\left(T_{\mathscr{R}}^{*}\right)$ определяет сюръективный гомоморфизм $\rho$ первой на вторую. Ядро этого гомоморфизма представляет собой нормальное замыкание в группе $\operatorname{FTP}\left(T^{*}\right)$ объединения подгрупп $R_{i}(0 \leq i \leq n)$ и потому содержится в $N$. Отсюда легко следует, что $N \rho \cap \operatorname{FTP}\left(T_{i}\right) \rho=1$ для каждого $i \geq 1$. Значит, естественный гомоморфизм группы $\mathrm{FTP}\left(T_{\mathscr{R}}^{*}\right)$ на фактор-группу $\operatorname{FTP}\left(T_{\mathscr{R}}^{*}\right) / N \rho$ действует инъективно на всех реберных подгруппах. Так как $\operatorname{ker} \rho \leq N$, то

$$
\operatorname{FTP}\left(T_{\mathscr{R}}^{*}\right) / N \rho=\operatorname{FTP}\left(T^{*}\right) \rho / N \rho \cong \operatorname{FTP}\left(T^{*}\right) / N \in \mathscr{C} .
$$

Поэтому группа $\operatorname{FTP}\left(T_{\mathscr{R}}^{*}\right) \mathscr{C}$-аппроксимируема в силу предложения 4 . Остается заметить, что согласно предложению $1 \rho$ продолжает естественный гомоморфизм $\operatorname{FTP}\left(T_{g}\right) \rightarrow \operatorname{FTP}\left(T_{g}\right) / R_{0}$, инъективный на группе $\operatorname{FTP}\left(T_{g}\right)$. Поэтому $g \rho \neq 1$ и $\rho$ может быть продолжен до гомоморфизма на группу из класса $\mathscr{C}$, переводящего $g$ в отличный от 1 элемент.

Предложение 6 [19, теорема 1]. Пусть $T$ - произвольное дерево и для любых $e \in E, v \in e$ подгруппа $H_{e, v}$ лежит в центре группы $G_{v}$. Тогда для каждой вершины $v \in V$ тождественное отображение образующих группы $G_{v}$ в группу $\operatorname{DTP}(T)$ продолжаемо до инъективного гомоморфизма и потому все группы $G_{v}$ $(v \in V)$ можно считать подгруппами группы $\operatorname{DTP}(T)$.

Если все группы $G_{v}(v \in V)$ не имеют кручения и для любых $e \in E, v \in e$ подгруппа $H_{e, v}$ изолирована в группе $G_{v}$, то группа $\operatorname{DTP}(T)$ не имеет кручения.

Предложение 7. Пусть $T$ - произвольное дерево и для любых $e \in E$, $v \in e$ подгруппа $H_{e, v}$ лежит в центре группы $G_{v}$. Тогда естественный гомоморфизм группы $\operatorname{FTP}(T)$ на группу $\operatorname{DTP}(T)$ действует тождественно на всех вершинных группах.

ДокАЗАтЕльство. В самом деле, требуемое утверждение следует из предложения 6 и коммутативности диаграммы

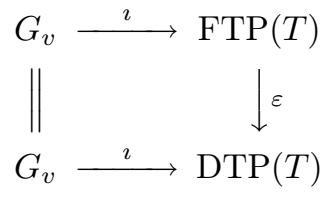

для каждой вершины $v \in V$ (здесь $\varepsilon$ обозначает естественный гомоморфизм группы $\mathrm{FTP}(T)$ на группу $\mathrm{DTP}(T), \imath$ - вложение, определяемое тождественным отображением образующих).

Обратимся к доказательству теорем 1, 2 и заметим, что первая из них сразу же вытекает из предложения 5. В самом деле, на роль отображения, фигурирующего в формулировке данного предложения, подходит естественный гомоморфизм $\mathrm{FTP}(T) \rightarrow \operatorname{DTP}(T):$ его инъективность на всех группах $H_{v}(v \in V)$ следует из предложения 7. 
Похожая схема рассуждений используется и для доказательства теоремы 2. Положим $R_{v}=\operatorname{ker} \sigma_{v}$ для каждой вершины $v \in V$ и рассмотрим граф $T_{\mathscr{R}}$, где $\mathscr{R}=\left\{R_{v} \mid v \in V\right\}$. Согласно предложению 1 тождественное отображение образующих группы $\mathrm{FTP}(T)$ в группу $\operatorname{FTP}\left(T_{\mathscr{R}}\right)$ определяет гомоморфизм $\rho$, продолжающий гомоморфизмы $\sigma_{v}(v \in V)$. Согласно предложению 7 гомоморфизм $\sigma=\rho \varepsilon$, где $\varepsilon: \operatorname{FTP}\left(T_{\mathscr{R}}\right) \rightarrow \operatorname{DTP}\left(T_{\mathscr{R}}\right)-$ естественный гомоморфизм, также можно считать продолжением отображений $\sigma_{v}(v \in V)$.

Группа $\operatorname{DTP}\left(T_{\mathscr{R}}\right)$ представляет собой гомоморфный образ прямого произведения конечного числа $\mathscr{C}$-групп $G_{v} / R_{v}(v \in V)$ и в силу условий, наложенных на класс $\mathscr{C}$, принадлежит данному классу. При этом если все группы $G_{v} / R_{v}$ $(v \in V)$ не имеют кручения и для любых $e \in E, v \in e$ подгруппа $H_{e, v} R_{v} / R_{v}$ изолирована в группе $G_{v} / R_{v}$, то по предложению $6 \operatorname{DTP}\left(T_{\mathscr{R}}\right)$ - группа без кручения. Следовательно, определенный выше гомоморфизм $\sigma$ является искомым отображением. Будучи продолжением гомоморфизмов $\sigma_{v}(v \in V)$, он инъективен на всех реберных подгруппах группы $\operatorname{FTP}(T)$. Поэтому ввиду предложения 4 данная группа аппроксимируется классом $\mathscr{C}$, а также классом всех $\mathscr{C}$-групп без кручения в случае, когда образ $\sigma$, группа $\operatorname{DTP}\left(T_{\mathscr{R}}\right)$, не имеет кручения и все группы $G_{v}(v \in V)$ аппроксимируются $\mathscr{C}$-группами без кручения.

\section{§ 4. Доказательства следствий 1 и 2}

ДокАЗАТЕЛЬСТвО СЛЕДСТВИЯ 1. Если ступени разрешимости групп $G_{v}$ $(v \in V)$ ограничены в совокупности, то их прямое произведение, а вместе с ним и группа $\mathrm{DTP}(T)$ разрешимы. Если в дополнение к этому все вершинные группы не имеют кручения, а реберные подгруппы изолированы в них, то в силу предложения 6 группа $\operatorname{DTP}(T)$ не имеет кручения. Если все группы $G_{v}(v \in V)$ являются периодическими $\pi$-группами конечного периода, то группа $\mathrm{DTP}(T)$ также оказывается разрешимой периодической $\pi$-группой конечного периода. Таким образом, все три утверждения следствия вытекают из теоремы 1.

ДоказАТЕЛЬСтво СЛЕДствия 2. Так как дерево $T$ конечно, в каждой вершинной группе $G_{v}$ содержится лишь конечное число реберных подгрупп. Поэтому, если все они конечны или имеют конечный ранг, тем же свойством обладает и подгруппа $H_{v}$.

Ввиду $\mathscr{C}$-аппроксимируемости группы $G_{v}$ для каждого неединичного элемента $h \in H_{v}$ найдется не содержащая этого элемента нормальная подгруппа $N_{h}$ такая, что $G_{v} / N_{h} \in \mathscr{C}$. По теореме Ремака фактор-группа $G_{v} / N_{v}$, где

$$
N_{v}=\bigcap_{h \in H_{v} \backslash\{1\}} N_{h},
$$

изоморфна подгруппе декартова произведения $P \mathscr{C}$-групп $G_{v} / N_{h}$. Если подгруппа $H_{v}$ конечна, то количество сомножителей в этом произведении также конечно и потому $P \in \mathscr{C}$. Значит, $G_{v} / N_{v} \in \mathscr{C}$ и $N_{v} \cap H_{v}=1$.

Если группа $G_{v}$ аппроксимируется $\mathscr{C}$-группами без кручения и подгруппа $H_{v}$ имеет конечный ранг, то существование такой нормальной подгруппы $N_{v}$ группы $G_{v}$, что $G_{v} / N_{v}-\mathscr{C}$-группа без кручения и $N_{v} \cap H_{v}=1$, обеспечивается предложением 11 из [16]. Наконец, если $G_{v} \in \mathscr{C}$, в качестве $N_{v}$ можно взять единичную подгруппу. Таким образом, для каждой вершины $v \in V$ существует гомоморфизм группы $G_{v}$ на $\mathscr{C}$-группу $G_{v} / N_{v}$, инъективный на подгруппе $H_{v}$, и группа $\mathrm{FTP}(T)$ является $\mathscr{C}$-аппроксимируемой в силу теоремы 2. 


\section{ЛИТЕРАТУРА}

1. Gruenberg K. W. Residual properties of infinite soluble groups // Proc. Lond. Math. Soc. 1957. V. 7. P. 29-62.

2. Sokolov E. V. A characterization of root classes of groups // Comm. Algebra. 2015. V. 43. P. 856-860.

3. Соколов Е. В. Некоторые аппроксимационные свойства обобщенных свободных произведений групп // Чебышевский сб. 2012. Т. 13, № 1. С. 143-149.

4. Kim G., Tang C. Y. On generalized free products of residually finite $p$-groups // J. Algebra. 1998. V. 201, N 1. P. 317-327.

5. Молдаванский Д. И. Финитная аппроксимируемость некоторых HNN-расширений групп // Вестн. Иван. гос. ун-та. Сер. Биология, Химия, Физика, Математика. 2002, № 3. С. 123-133.

6. Молдаванский Д. И. Аппроксимируемость конечными $p$-группами некоторых HNN-pacширений групп // Вестн. Иван. гос. ун-та. Сер. Биология, Химия, Физика, Математика. 2003. № 3. C. 102-116.

7. Aschenbrenner M., Friedl S. A criterion for HNN extensions of finite $p$-groups to be residually p // J. Pure Appl. Algebra. 2011. V. 215. P. 2280-2289.

8. Туманова $E$. А. Об аппроксимируемости конечными $\pi$-группами обобщенных свободных произведений групп // Мат. заметки. 2014. Т. 95, № 4. С. 605-614.

9. Азаров Д. Н. Аппроксимируемость конечными $p$-группами обобщенных свободных произведений групп // Изв. вузов. Математика. 2017. № 5. С. 3-10.

10. Азаров Д. Н., Тьеджо Д. Об аппроксимируемости свободного произведения групп с объединенной подгруппой корневым классом групп // Науч. тр. Иван. гос. ун-та. Математика. 2002. № 5. С. 6-10.

11. Азаров Д. Н., Туманова Е. А. Об аппроксимируемости обобщенных свободных произведений групп корневыми классами // Науч. тр. Иван. гос. ун-та. Математика. 2008. № 6. C. $29-42$.

12. Tieudjo D. On root-class residuality of some free constructions // JP J. Algebra, Number Theory Appl. 2010. V. 18, N 2. P. 125-143.

13. Туманова Е. А. Об аппроксимируемости корневыми классами $\mathrm{HNN}$-расширений групп // Модел. и анализ информ. систем. 2014. Т. 21, № 4. С. 148-180.

14. Гольцов Д. В. Аппроксимируемость HNN-расширения с центральными связанными подгруппами корневым классом групп // Мат. заметки. 2015. Т. 97, № 5. С. 665-669.

15. Туманова E. А. Об аппроксимируемости корневыми классами групп обобщенных свободных произведений с нормальным объединением // Изв. вузов. Математика. 2015. № 10. C. $27-44$.

16. Соколов E. В., Туманова E. А. Достаточные условия аппроксимируемости некоторых обобщенных свободных произведений корневыми классами групп // Сиб. мат. журн. 2016. T. 57, № 1. C. 171-185.

17. Соколов E. B., Туманова E. А. Аппроксимируемость корневыми классами HNN-расширений с центральными циклическими связанными подгруппами // Мат. заметки. 2017. Т. 102 , № 4. C. $597-612$.

18. Туманова E. А. Аппроксимируемость корневыми классами групп древесных произведений с объединенными ретрактами // Сиб. мат. журн. 2019. Т. 60, № 4. С. 891-906.

19. Соколов E. В., Туманова Е. А. Обобщенные прямые произведения групп и их применение к изучению аппроксимируемости свободных конструкций групп // Алгебра и логика. 2019. T. 58, № 6. C. $720-740$.

20. Sokolov E. V., Tumanova E. A. To the question of the root-class residuality of free constructions of groups // Lobachevskii J. Math. 2020. V. 41, N 2. P. 260-272.

21. Соколов E. В., Туманова E. А. Об аппроксимируемости корневыми классами некоторых свободных произведений групп с нормальными объединенными подгруппами // Изв. вузов. Математика. 2020. № 3. С. 48-63.

22. Kim G., Tang C. Y. A criterion for the conjugacy separability of amalgamated free products of conjugacy separable groups // J. Algebra. 1996. V. 184, N 3. P. 1052-1072.

23. Kim G. On the residual finiteness of fundamental groups of graphs of certain groups // J. Korean Math. Soc. 2004. V. 41, N 5. P. 913-920.

24. Raptis E., Talelli O., Varsos D. On the conjugacy separability of certain graphs of groups // J. Algebra. 1998. V. 199, N 1. P. 327-336. 
25. Kim G., Tang C. Y. Separability properties of certain tree products of groups // J. Algebra. 2002. V. 251, N 1. P. 323-349.

26. Varsos $D$. The residual nilpotence of the fundamental group of certain graphs of groups // Houston J. Math. 1996. V. 22, N 2. P. 233-248.

27. Kahrobaei $D$. On residual solvability of generalized free products of finitely generated nilpotent groups // Comm. Algebra. 2011. V. 39, N 2. P. 647-656

28. Kahrobaei D., Majewicz $S$. On the residual solvability of generalized free products of solvable groups // DMTCS. 2012. V. 13, N 4. P. 45-50.

29. Karras A., Solitar D. The subgroups of a free products of two groups with an amalgamated subgroup // Trans. Amer. Math. Soc. 1970. V. 150. P. 227-255.

Поступила в редакиию 6 ноября 2019 г.

После доработки 26 февраля 2020 г.

Принята $к$ публикации 8 апреля 2020 2.

Соколов Евгений Викторович, Туманова Елена Александровна

Ивановский государственный университет,

ул. Ермака, 39, Иваново 153025

ev-sokolov@yandex.ru, helenfog@bk.ru 